\title{
Familial lethal cardiomyopathy with mental retardation and scapuloperoneal muscular dystrophy
}

\author{
BERTA BERGIA,* HARLEY D SYBERS, $\dagger$ IAN J BUTLER* \\ From the Departments of Neurology* and Pathology, $\dagger$ Medical School, University of Texas Health Science \\ Center at Houston, USA
}

\begin{abstract}
SUMMARY A family is described with a neuromuscular disorder characterised by possible X-linked recessive inheritance, a benign, slowly progessive muscular dystrophy with predominant humeroperoneal distribution and lack of contractures or pseudohypertrophy, central nervous system involvement, myopia and lethal cardiomyopathy. The possibility of cardiac transplant as life-saving therapy is suggested.
\end{abstract}

In a number of familial disorders affecting skeletal muscle there are associated defects in cardiac and central nervous system functions. Such associations are apparent in Duchenne and Becker type muscular dystrophy and myotonic dystrophy. Disorders of glycogen metabolism, such as acid-maltase deficiency, or disturbed carnitine metabolism, may also have defective function of muscle, heart and brain.

In this family in which two sisters had affected male children with a distinct neuromuscular disorder, mental retardation was observed early in the first decade followed by a relatively benign dystrophic process affecting skeletal muscle. Two of the three affected boys died of hypertrophic cardiomyopathy late in the second decade.

\section{Case reports}

(See pedigree, fig 1)

\section{Patient III-3}

A 17 year old male with mild motor incoordination developed normally until 6 years of age. During evaluation for a learning disability he was diagnosed as mentally retarded and placed in special education. Despite significant problems with coordination and balance he was not evaluated until age 16 years following hospitalisation for a syncopal episode. A chest radiograph showed cardiomegaly (fig $2 a$ ) and a 2-D echocardiogram revealed hypertrophic cardiomyopathy with outflow obstruction during systole. An electrocardiogram (ECG) showed a left bundle branch block, fre-

Address for reprint requests: Professor Ian J Butler, Department of Neurology, University of Texas Health Science Center, PO Box 20708, Houston, Texas 77225, USA.

Received 28 January 1986. Accepted 8 March 1986 quent premature ventricular contractions and occasional premature supraventricular complexes (fig 2b). Laboratory studies included elevated levels of creatine kinase (CK) to $840 \mathrm{U} / 1$ (normal 2-235 U/1), lactic acid dehydrogenase (LDH) $810 \mathrm{U} / 1$ (normal 100-190 U/1), aspartate aminotransferase (SGOT) $480 \mathrm{U} / 1$ (normal 22-47 U/1), and alanine aminotransferase (SGPT) $230 \mathrm{U} / 1$ (normal 0-35 U/1). At age 17 years he was evaluated for progressive dyspnoea on exertion. Physical examination revealed missed heart beats, cardiomegaly, severe mental retardation and marked myopia. Further examination at this medical centre showed wasting of distal muscle groups, positive Gower's manoeuvre and predominant humero-peroneal distribution of muscle weakness. Tendon reflexes were present and myotonia was absent. Serum carnitine, serum and urine aminoacids, urine organic acids and urine oligosaccharide chromatography were normal. Chromosomal analysis was normal. Lysosomal enzymes in white blood cells and cultured skin fibroblasts were normal. Phosphorylase in cultured skin fibroblasts and muscle, $\alpha$-glucosidase in cultured skin fibroblasts and white blood cells debrancher enzyme activity and glycogen content in muscle were all normal. Conjunctival biopsy did not indicate a storage disease.

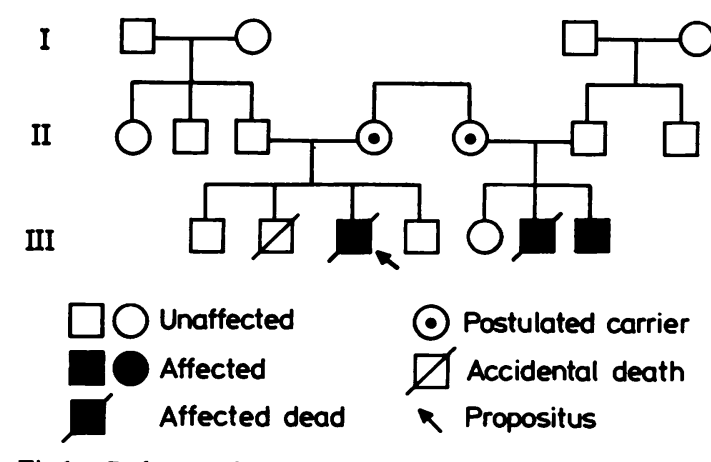

Fig 1 Pedigree of the family. 

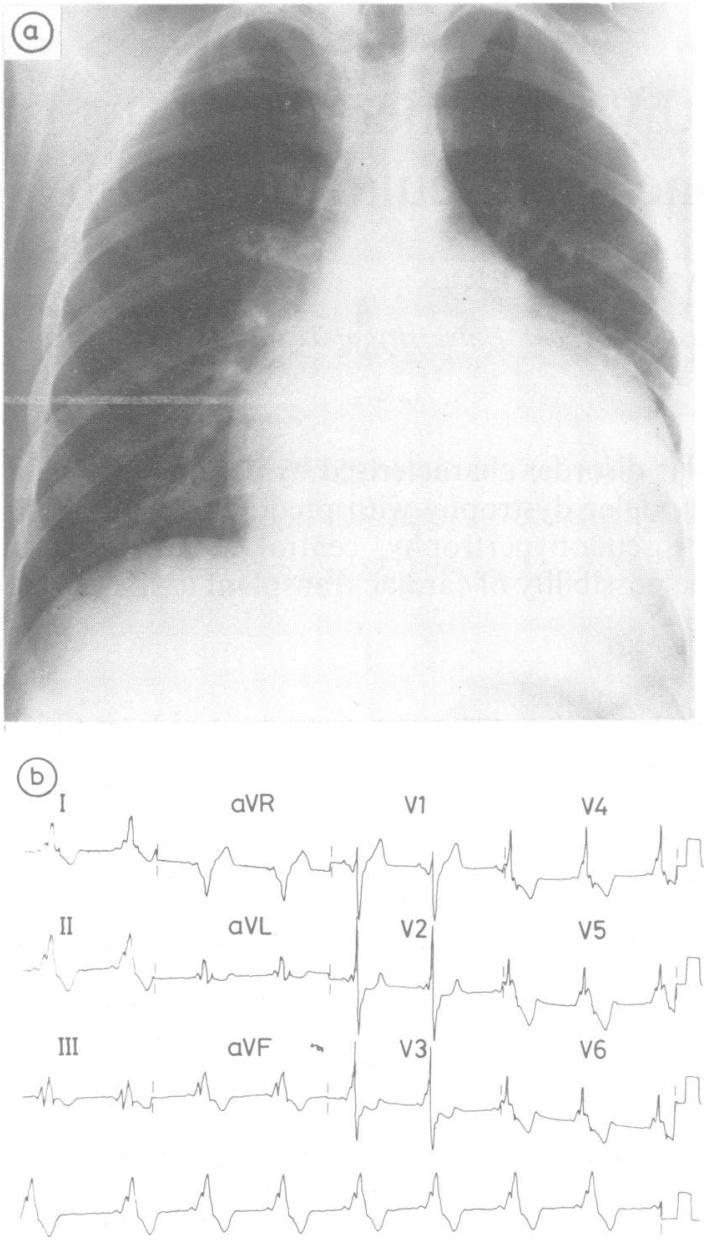

Fig 2 (a) Chest radiograph of patient III-3 showed cardiomegaly. (b) Electrocardiogram demonstrated left bundle branch block.

Electrophysiological studies showed an abnormal electroencephalogram (EEG) with runs of polymorphic delta activity in the right posterior temporal area superimposed upon a generally slow and disorganised background. Brainstem auditory evoked responses were abnormal with mild disruption at the rostral pontine region bilaterally and there was mild prolongation of central conduction of median nerve somatosensory evoked responses. Visual evoked responses were at the upper limits of normal bilaterally. Nerve conduction velocities of sensory and motor nerves in the right upper and lower extremities were normal. Needle electromyography showed a small to moderate number of fibrillations and positive sharp waves mainly in the leg muscles. Motor units were of mixed myopathic and neuropathic type with a suggestion of increased recruitment. These results, in conjunction with normal nerve conduction studies, indicated a predominantly myopathic process.

A muscle biopsy specimen from the left quadriceps revealed moderate variations in fibre size with frequent internal nuclei and occasional fibre splitting (fig 3a). Occasionally a necrotic fibre undergoing phagocytosis was seen but the remaining fibres did not show marked hypertrophy. Only a slight increase in perimysial fibrosis and fatty infiltration was present and there was no increase in endomysial connective tissue. Electron microscopy confirmed the light microscopic findings and revealed a modest increase in glycogen granules and variation in mitochondrial size and shape (fig $3 \mathrm{~b}$ ). The findings were consistent with a dystrophic process.

Four months later an echocardiogram showed marked deterioration with an essentially akinetic myocardium and pericardial effusion and shortly thereafter he had a cardiorespiratory arrest and died.

At necropsy the heart was greatly enlarged $(1420 \mathrm{~g})$ with hypertrophy and dilatation of all chambers. There were diffuse focal scars scattered transmurally throughout the ventricular myocardium of both chambers. In addition to increased subendocardial fibrosis, patchy subepicardial fibrosis was also present. Microscopic examination confirmed the scattered focal nature of the lesions which ranged from focal scars to dense collagen to more recent foci of organising granulation tissue (fig 3c). The myocytes adjacent to the lesions revealed large prominent hyperchromatic nuclei characteristic of hypertrophy. Extensive cytoplasmic vacuolation and fatty change were present in many of the myocytes. Evidence of congestive heart failure was present in the lungs (congestion and oedema), liver (centrilobular congestion and necrosis), and spleen. The brain was grossly normal and microscopy was not performed.

\section{Patient III-6}

A maternal male cousin of case III-3 had normal development until onset of intellectual deterioration at age 5 years. He was severely retarded in school and unable to engage in physical activity because of shortness of breath and an awkward gait. Muscle enzymes in serum were elevated although precise values were not available. He died at age 21 years of presumed cardiac dysrhythmia and at necropsy his heart showed evidence of chronic myocarditis. There was marked concentric hypertrophy of both right and left ventricular systems and interstitial fibrosis. No coronary artery or cardiac valve abnormalities were noted. The liver had normal hepatic architecture with acute congestion. The brain was unremarkable on gross inspection, and no microscopy was obtained.

\section{Patient III-7}

A 17 year old male was evaluated at age 13 years because of the sudden death in a male sibling (patient III-6). His perinatal and developmental history were normal until at age 5 years his mother noted a decline in his intellectual abilities. He tired easily, had dyspnoea on exertion, and occasional episodes of dizziness but no syncope. At age 13 he had moderate mental retardation with a global IQ of 65 , severe myopia, mild scapular winging but normal muscle tone and mass without weakness or hypertrophy. Reflexes were symmetrically present and his neurological examination was otherwise unremarkable. Cardiac evaluation, including 24-hour Holter monitoring, showed several runs of supra- 

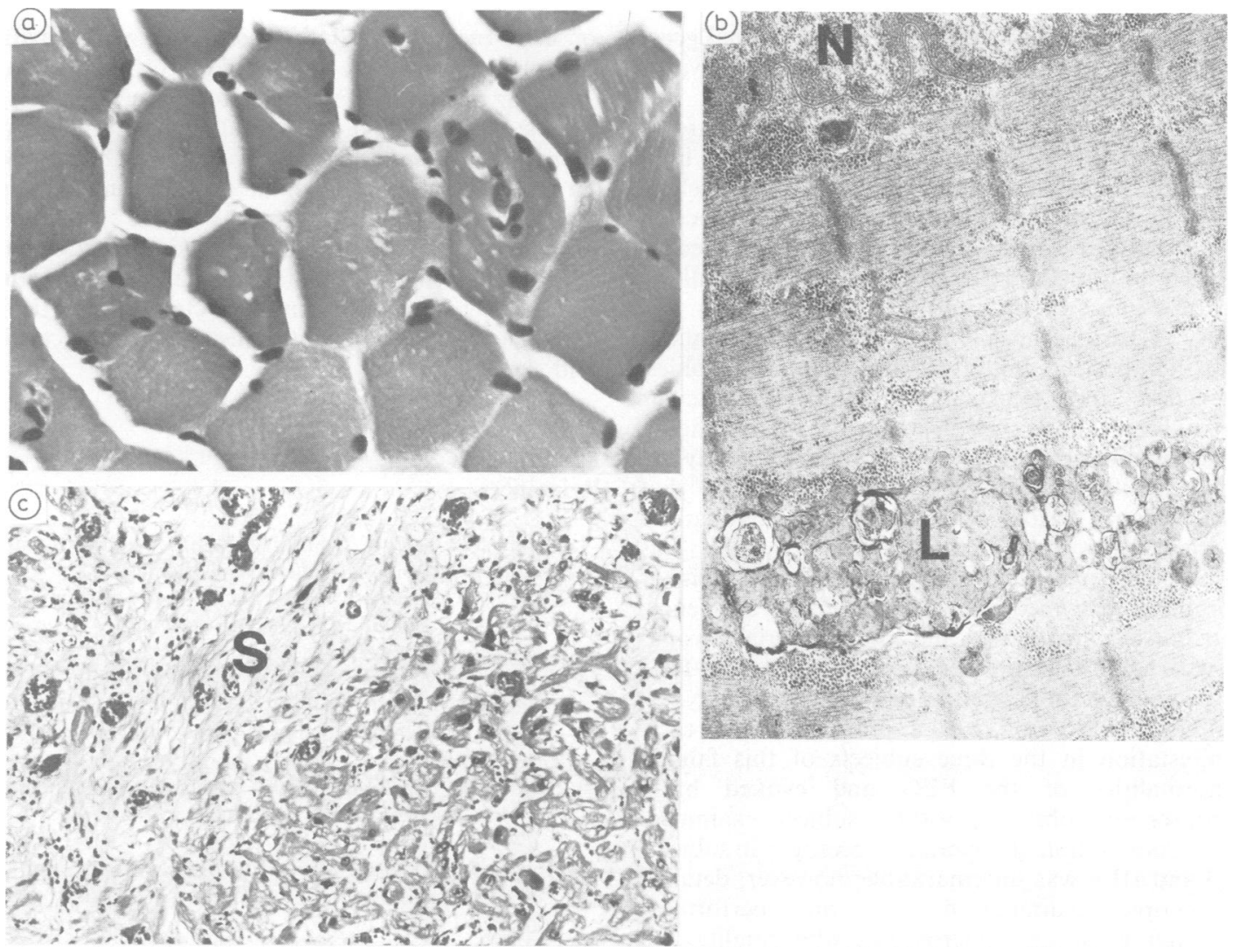

Fig 3 (a) Biopsy of left quadriceps revealed variation in fibre size and shape. Internal nuclei were frequent and occasionally multiple. Fibre splitting was occasionally seen. There was no increase in endomysial fibrous tissue $(H \& E \times 1900)$. (b) Electron micrograph of quadriceps revealed a generally normal ultrastructure except for an increased number of secondary lysosomes containing segments of focally necrotic fibres. Nucleus $(N) L y s o s o m e(L)$ $(\times 19,000)$. (c) Micrography of left ventricle illustrated diffuse increase in interstitial connective tissue separating myocytes, and focal areas of highly vascular scar tissue(s) infiltrated by lymphocytes and macrophages. Many myocytes were hypertrophic and many have vacuolated cytoplasm. ( $H \& E \times 200)$.

ventricular tachycardia, occasional premature ventricular contractions and a high nodal rhythm. M-mode echocardiography showed concentric left ventricular hypertrophy and an enlarged right ventricle. EKG showed right bundle branch block with left ventricular strain. Cardiomegaly with left ventricular prominence was present on radiographs and computed tomographic scan of the brain was normal. Serum levels of CK $(840 \mathrm{U} / 1 ; 100 \% \mathrm{MM}$ isoenzyme), SGPT (186 U/1), SGOT (301 U/1) and alkaline phosphatase $(263 \mathrm{U} / \mathrm{l})$ were elevated.

\section{Patient II-4}

The mother of patient III/3 had a cardiac evaluation at age 43 years and was taking digoxin for heart failure. 2D-echocardiogram showed marked dilatation of the left ventricle with diminished function. Serum muscle enzymes, including CK, aldolase, SGOT and LDH were all normal.

Further evaluation of other family members has been difficult since the surviving affected male (III-7) and his parents live in another state. Two brothers of patient III-3 are clinically unaffected by history.

\section{Discussion}

Although in this family the inheritance pattern is consistent with an $\mathrm{X}$-linked recessive gene defect, the family history was limited since both mothers were adopted and dominant inheritance with variable expressivity is possible. The clinical features were similar in all three boys with onset of mental deterioration at age 5 years followed by a slowly progressive and relatively mild myopathy. During the mid-teens years there was onset of a cardiomyopathy with progression to death in two subjects within a few years. Cardiac transplantation should be considered in the surviving male. Of interest the mother of patient 
III-3, and a presumed carrier for the abnormal gene, had evidence of a cardiomyopathy without elevated serum muscle enzymes.

Abnormal cardiac function in the form of cardiac conduction defects or a cardiomyopathy have been observed in various dystrophic disorders of skeletal muscle. $^{1-5}$ Detailed evaluations of cardiac function by prolonged EKG monitoring, 2-D echocardiography, electrophysiologic studies of bundle of His functions, quantitation of cardiac output and biopsy of cardiac muscle have shown abnormalities in a number of inherited muscular dystrophies. Duchenne and Becker-type X-linked muscular dystrophies, limb girdle, facioscapulohumeral, myotonic muscular dystrophy and more recently the Emery-Dreifuss syndrome ${ }^{125}$ are disorders that have defective cardiac function. Other myopathies associated with cardiomyopathy, such as $\alpha$-glucosidase deficiency, Type III glycogenosis or debrancher enzyme deficiency, carnitine deficiency, centronuclear myopathy, and mitochondrial myopathy with lactic acidosis, were excluded in patient III-3 by specific metabolic studies or muscle histology.

Mental retardation was a prominent and early manifestation in the three subjects of this family. Abnormalities of the EEG and evoked brain responses were observed in those subjects examined. Gross examination of the brain at necropsy in subjects III-3 and III-6 was unremarkable; however, detailed microscopic examination was not performed. Although gross and microscopic abnormalities of brain in 12 subjects with Duchenne type muscular dystrophy have been demonstrated, a later study failed to show significant CNS abnormalities as a cause for the mild to moderate mental retardation that may be present. ${ }^{6}$ Mental retardation can be a prominent early feature in myotonic dystrophy and may be a presenting manifestation. Reduced intelligence was present in two members of a family of five males with a slowly progressive myopathy in the scapuloperoneal distribution and associated with contractures, colour-blindness and cardiomyopathy in adult-life. ${ }^{7}$ In the recessive form of congenital muscular dystrophy of Fukuyama there is severe, early onset of mental retardation associated with obvious malformations of the brain. ${ }^{8}$ Two brothers with a vacuolar myopathy, excessive storage of glycogen in muscle, cardiomyopathy, mental retardation and normal acid maltase activity were described. ${ }^{9}$ Two unrelated boys with a similar clinical condition and normal acid maltase activity were previously described. ${ }^{10}$ Whether these cases and our family have a similar defect will depend on further metabolic studies to determine a common factor in electrically excitable tissues of the central nervous system, cardiac and skeletal muscle.

\section{References}

1 Dickey RP, Ziter FA, Smith RA. Emery-Dreifuss muscular dystrophy. J Pediatr 1984;104:555-9.

2 Emery AE, Dreifuss FE. Unusual type of benign Xlinked muscular dystrophy. $J$ Neurol Neurosurg Psychiatry 1966;29:338-41.

3 Fenichel GM, Sul YC, Kilroy AW, Blouin R; An autosomal-dominant dystrophy with humeropelvic distribution and cardiomyopathy. Neurology 1982; 32:1399-401.

4 Perloff JK. Cardiomyopathy associated with heredofamilial neuromyopathic diseases. Mod Concepts of Cardiovascular disease 1971;40:23-6.

5 Rowland LP, Fetell M, Olarte M, Hays A, Singh N, Wanat FE. Emery-Dreifuss muscular dystrophy. Ann Neurol 1979;5:111-7.

6 Dubowitz V, Crome L. The central nervous system in Duchenne muscular dystrophy. Brain 1969;92:805-8.

7 Thomas PK, Calne DB, Elliot CF. X-linked scapuloperoneal syndrome. J Neurol Neurosurg Psychiatry 1972;35:208-15.

8 Fukuyama $Y$, Kawazura $M$, Haruna $H$. A peculiar form of congenital progressive muscular dystrophy. Paediatr Univ Tokyo 1960;4:5-8.

9 Riggs JE, Schochet SS, Gutmann L, Shanske S, Neal WA, DiMauro S. Lysosomal glycogen storage disease without acid maltase deficiency. Neurology 1983; 33:873-7.

10 Danon MJ, Oh SJ, DiMauro S, etal. Lysosomal glycogen storage disease with normal acid maltase. Neurology 1981;31:51-7. 